\title{
Dirac-Point Shift by Carrier Injection Barrier in Graphene Field-Effect Transistor Operation at Room Temperature
}

Sungsik Lee, Arokia Nathan, Jack A. Alexander-Webber, Philipp Braeuninger-Weimer, Abhay A. Sagade, Haichang Lu, David G. Hasko, John Robertson, and Stephan Hofmann

ACS Appl. Mater. Interfaces, Just Accepted Manuscript • DOI: 10.1021/acsami.8b02294 • Publication Date (Web): 20 Mar 2018

Downloaded from http://pubs.acs.org on March 21, 2018

\section{Just Accepted}

"Just Accepted" manuscripts have been peer-reviewed and accepted for publication. They are posted online prior to technical editing, formatting for publication and author proofing. The American Chemical Society provides "Just Accepted" as a service to the research community to expedite the dissemination of scientific material as soon as possible after acceptance. "Just Accepted" manuscripts appear in full in PDF format accompanied by an HTML abstract. "Just Accepted" manuscripts have been fully peer reviewed, but should not be considered the official version of record. They are citable by the Digital Object Identifier (DOI®). "Just Accepted" is an optional service offered to authors. Therefore, the "Just Accepted" Web site may not include all articles that will be published in the journal. After a manuscript is technically edited and formatted, it will be removed from the "Just Accepted" Web site and published as an ASAP article. Note that technical editing may introduce minor changes to the manuscript text and/or graphics which could affect content, and all legal disclaimers and ethical guidelines that apply to the journal pertain. ACS cannot be held responsible for errors or consequences arising from the use of information contained in these "Just Accepted" manuscripts. 


\title{
Dirac-Point Shift by Carrier Injection Barrier in
}

\section{Graphene Field-Effect Transistor Operation at Room}

\section{Temperature}

Sungsik Lee ${ }^{1,2}$, Arokia Nathan ${ }^{1 *}$, Jack Alexander-Webber ${ }^{1}$, Philipp Braeuninger-Weimer ${ }^{\text {, }}$, Abhay A. Sagade ${ }^{1,3}$, Haichang Lu ${ }^{1}$, David Hasko ${ }^{1}$, John Robertson ${ }^{1}$, Stephan Hofmann ${ }^{1}$

1. Electrical Engineering Division, Department of Engineering, University of Cambridge, 9 JJ Thomson Avenue, Cambridge, CB3 0FA, United Kingdom

2. Currently at Department of Electronics Engineering, Pusan National University, Pusan 46241, Republic of Korea.

3. Currently at SRM Research Institute and Department of Physics, SRM Institute of Science and Technology, Kattankulathur 603 203, Tamil Nadu, India

\author{
KEYWORDS \\ Graphene field effect transistor, Dirac point, Fermi velocity, Asymmetric injection, Intrinsic \\ carrier mobility, Short-range scattering
}

\begin{abstract}
A positive shift in the Dirac-point in graphene field-effect transistors was observed with Halleffect coupled with Kelvin-probe measurements at room temperature. This shift can be explained by the asymmetrical behaviour of the contact resistance by virtue of the electron injection barrier at the source contact. As an outcome, an intrinsic resistance is given to allow a retrieval of an intrinsic carrier mobility found to be decreased with increasing gate bias, suggesting the dominance of short-range scattering in a single layer graphene field-effect transistor. These results analytically correlate the field-effect parameters with intrinsic graphene properties.
\end{abstract}


Ever since graphene was identified as a promising electronic material for newly emerging applications, there have been numerous studies on its material properties and associated device performance ${ }^{1-8}$. This has led to a study of new device architectures, and in particular, attempts to identify the role of contact materials with the graphene layer ${ }^{9-11}$, suggesting that contact properties significantly limit the performance of graphene devices. At the same time, there has been equally important emphasis on the study of the intrinsic electronic properties of "bulk" graphene ${ }^{4-6}$. For example, it has been reported that the Dirac cones of a suspended graphene layer are reshaped by carrier interaction effects associated with the carrier density in graphene ${ }^{8,12}$. However, when used as the channel in a field-effect device, it is important to understand how the graphene material properties correlate with device characteristics. On this, it has been reported that the Dirac point can be shifted due to contact properties at the source and drain electrodes, which was observed either by a four-point probe measurement at low temperatures ${ }^{13}$, e.g. $60 \mathrm{~K}$, or by employing different work-function metals for the source and drain electrodes 14,15. Although there have been previous studies on the effects of contacts on graphene fieldeffect devices, these have not been used to understand how the intrinsic properties, such as contact effects, Dirac point, carrier density, and intrinsic carrier mobility are influenced at room temperature by an orthogonal electric field, and their inter-relation in a field-effect structure. This constitutes the focus of the investigations presented in this work.

Using a combination of Hall-effect and Kelvin probe measurements at room temperature, we examine the symmetry in behaviour of the intrinsic resistance as a function of gate bias, retrieved from the incongruity in the contact and extrinsic resistances of the device. The resulting positive shift in the extrinsic Dirac point, from that of the intrinsic counterpart is explained by the asymmetrical behaviour of the contact resistance as a function of gate bias, suggesting the 

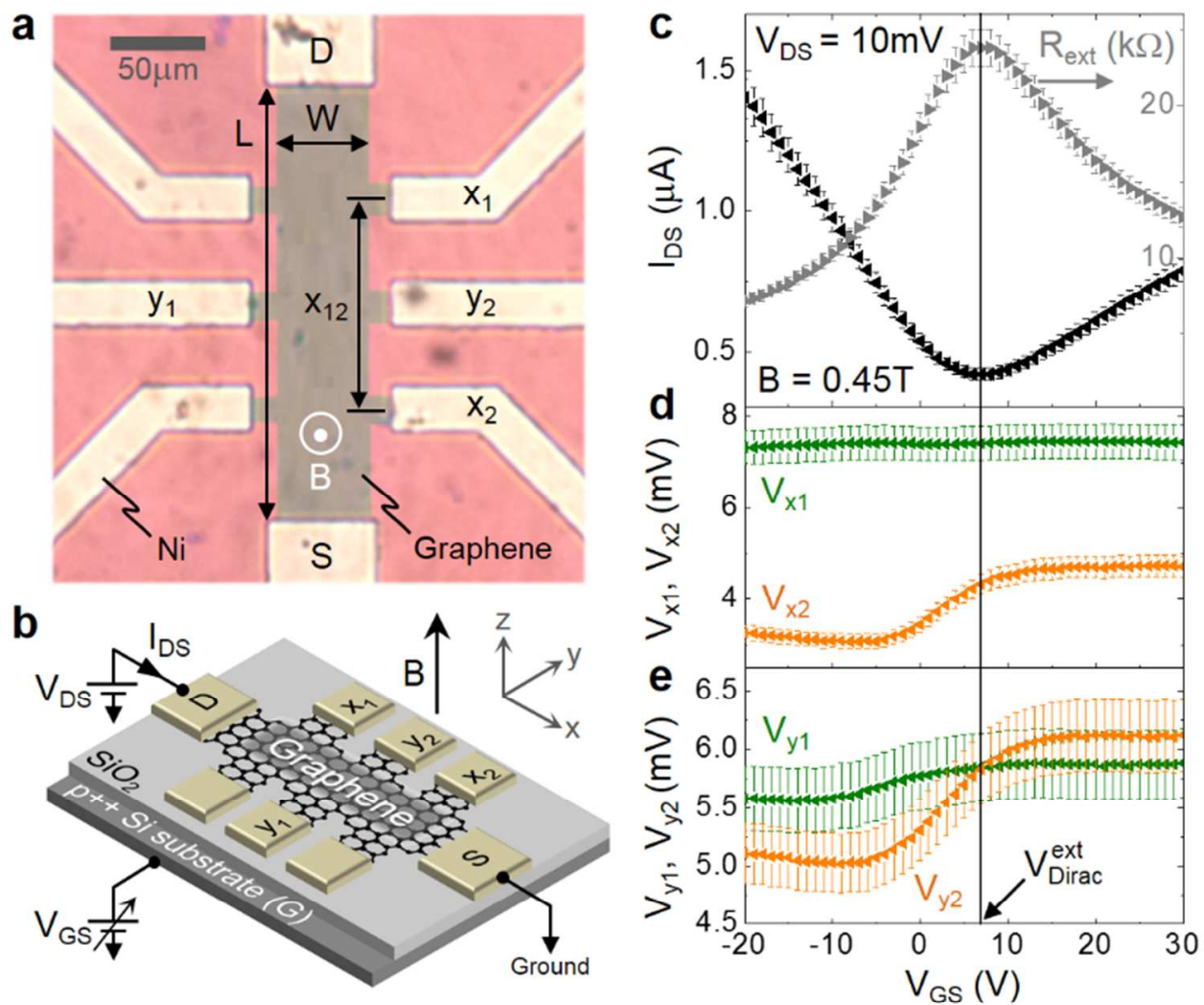

Figure 1. Hall-effect and Kelvin probe measurements on the graphene field-effect transistor: (a) Micro-photo of the fabricated structure and its (b) 3-D view along with the electrical measurement set-up. Here, the channel length (L), width (W), and vertical separation $\left(\mathrm{x}_{12}\right)$ are $250 \mu \mathrm{m}, 50 \mu \mathrm{m}$, and $120 \mu \mathrm{m}$, respectively. (c) Measured drain current $\left(\mathrm{I}_{\mathrm{DS}}\right)$ at $\mathrm{V}_{\mathrm{DS}}=$ $10 \mathrm{mV}$ and the equivalent resistance $\left(\mathrm{R}_{\mathrm{ext}}=\mathrm{V}_{\mathrm{DS}} / \mathrm{I}_{\mathrm{DS}}\right)$ as a function of $\mathrm{V}_{\mathrm{GS}}$ for $\mathrm{B}=0.45 \mathrm{~T}$. (d) Measured $V_{\mathrm{x} 1}$ and $\mathrm{V}_{\mathrm{x} 2}$ as a function of $\mathrm{V}_{\mathrm{GS}}$, and (e) measured $\mathrm{V}_{\mathrm{y} 1}$ and $\mathrm{V}_{\mathrm{y} 2}$ as a function of $\mathrm{V}_{\mathrm{GS}}$. Here, the measured data in Figs. $1 \mathrm{c}$ to e have a $5 \%$ error-bar.

presence of an injection barrier for electrons by virtue of the higher work-function of the nickel (source contact) electrode compared to the graphene layer. In addition, Dirac voltage is observed to be the common intercept for the linear variation in electron and hole carrier densities. From the extracted intrinsic resistance and carrier density, the intrinsic carrier mobility is retrieved showing its decrease with increasing gate bias. This suggests dominance of short-range scattering, the rate of which can increase as more carriers are induced by a higher gate bias, and has been observed previously in single layer graphene. These results provide analytical insight 
into the underlying physics across the field-dependent intrinsic parameters, while maintaining a consistency with earlier reports.

Fig.1 shows representative results from Hall-effect coupled with Kelvin probe measurements of a graphene field-effect transistor; the detailed fabrication process has been published elsewhere ${ }^{17,18}$, and can also be found in the Supporting Information. The photomicrograph of the measured test structure is shown in Fig.1a. The electrodes on the x-axis are used to measure the internal voltage drops and that on the y-axis are for measuring the Hallvoltage $\left(\mathrm{V}_{\mathrm{H}}\right)$. The source $(\mathrm{S})$ and drain (D) electrodes are on an oxidized high-doped silicon wafer which serves as the gate $(\mathrm{G})$. The gate bias $\left(\mathrm{V}_{\mathrm{GS}}\right)$ is swept at a constant drain bias $\left(\mathrm{V}_{\mathrm{DS}}\right)$ in the presence of a magnetic field (B) applied orthogonally (i.e. along the z-axis) and uniformly over the graphene layer. Along with this, a drain current $\left(\mathrm{I}_{\mathrm{DS}}\right)$ at a small drain bias $\left(\mathrm{V}_{\mathrm{DS}}\right)$ and the equivalent extrinsic resistance (i.e. $R_{\text {ext }}=V_{D S} / I_{D S}$ ) are measured as a function of $V_{G S}$ for $B=$ $0.45 \mathrm{~T}$, as seen in Fig.1c. Here, $\mathrm{V}_{\mathrm{DS}}$ is fixed at $10 \mathrm{mV}$ which is sufficiently smaller than the gate bias and thermal voltage (about $26 \mathrm{mV}$ at room temperature, i.e. $300 \mathrm{~K}$ ) to satisfy the gradual channel approximation ${ }^{19}$. In addition, the voltage levels $\left(\mathrm{V}_{\mathrm{x} 1}, \mathrm{~V}_{\mathrm{x} 2}\right)$ at the nodes of $\mathrm{x}_{1}$ and $\mathrm{x}_{2}$ are measured while measuring the voltage levels $\left(\mathrm{V}_{\mathrm{y} 1}, \mathrm{~V}_{\mathrm{y} 2}\right)$ at the nodes $\mathrm{y}_{1}$ and $\mathrm{y}_{2}$ as a function of $\mathrm{V}_{\mathrm{GS}}$, respectively (see Figs.1d and e). Here, the extrinsic Dirac voltage (i.e. $V_{\text {Dirac }}^{\text {ext }}$ ) is retrieved at the minimum point of $I_{D S}$ (i.e. the peak of $R_{e x t}$ ), which is consistent with $V_{G S}$ at which $V_{y 1}=V_{y 2}$. Note that the extrinsic Dirac voltage can be shifted due to atmospheric absorption ${ }^{19}$.

Based on the experimental results discussed with Fig.1, the gate voltage-dependence of the intrinsic parameters of the graphene device is shown in Fig.2. Firstly, the contact resistance $\left(R_{C}\right)$ is found from the difference between $R_{e x t}$ and the intrinsic resistance $\left(R_{\text {int }}\right)$, i.e. $R_{C}=R_{\text {ext }}$ $\mathrm{R}_{\mathrm{int}}$, which are as functions of $\mathrm{V}_{\mathrm{GS}}$, respectively. Here, $\mathrm{R}_{\mathrm{int}}$ is described by the following relation, 

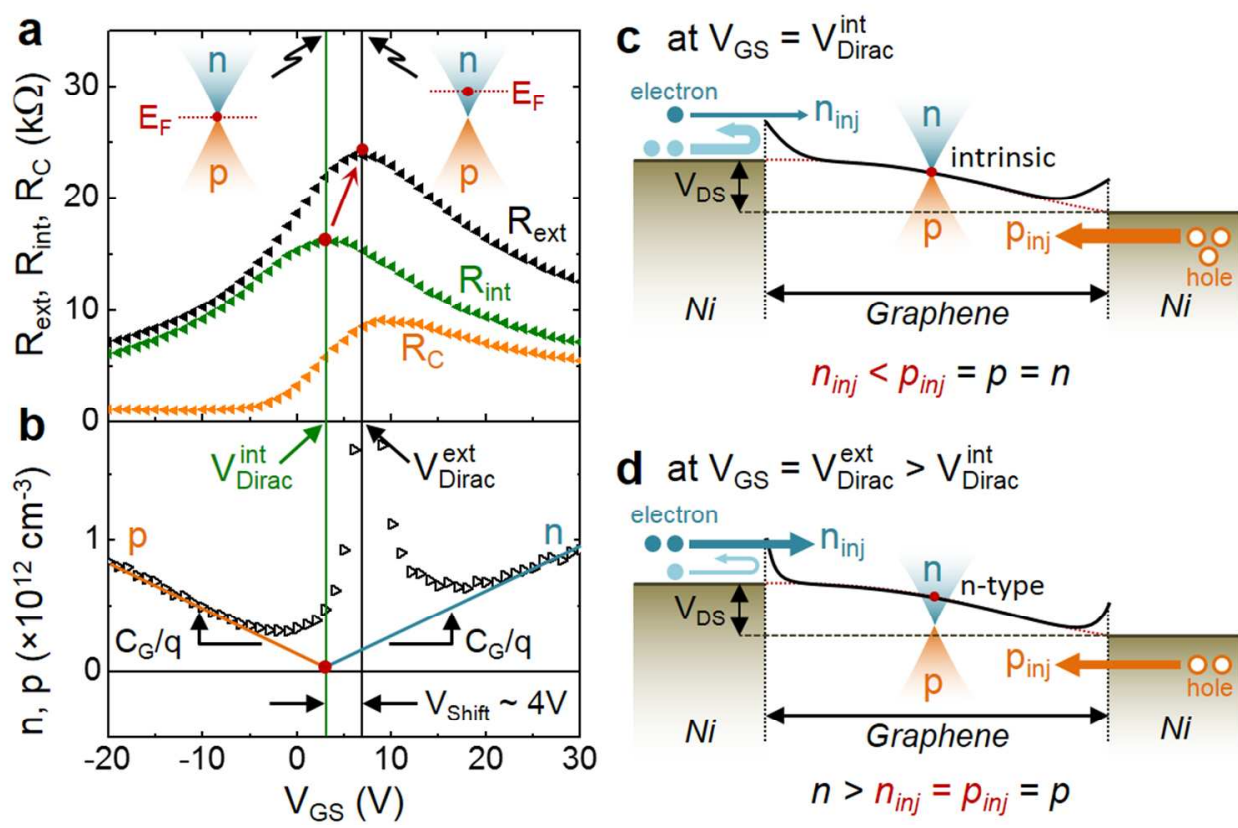

Figure 2. Gate voltage-dependence of intrinsic parameters of the graphene transistor and underlying band diagram illustrating electron and hole injection: (a) Retrieved $R_{C}$ from the difference between $R_{\text {ext }}$ and $R_{\text {int }}$ (b) Extracted concentrations of the free electrons (n) and holes (p) as a function of $\mathrm{V}_{\mathrm{GS}}$, respectively. (c) Conceptual band diagram to describe the blocking of electron injection at $V_{G S}=V_{\text {Dirac }}^{\text {int }}$. (d) Conceptual band diagram at $V_{G S}=V_{\text {Dirac }}^{\text {ext }}$.

$$
R_{i n t}=\left(\frac{V_{x 1}-V_{x 2}}{I_{D S}}\right) \frac{L}{x_{12}},
$$

where $\mathrm{x}_{12}$ is the distance between the electrodes $\mathrm{x}_{1}$ and $\mathrm{x}_{2}$, as indicated in Fig.1a. It is observed that the peak point of $\mathrm{R}_{\text {ext }}\left(\right.$ i.e. extrinsic Dirac voltage, $V_{\text {Dirac }}^{\text {ext }}$ ) is right-shifted by 4 volts from that of $\mathrm{R}_{\text {int }}$ (i.e. intrinsic Dirac voltage, $V_{\text {Dirac }}^{\text {int }}$ ) (see Figs.2a and $2 \mathrm{~b}$ ). This suggests the presence of an electron injection barrier, corresponding to the asymmetrical characteristics of $R_{C}$ as a function of $\mathrm{V}_{\mathrm{GS}}$, as can be seen in Fig.2a. Note that, if there was a hole injection barrier, this would be shifted the other way. Here, the sign of $V_{\text {Dirac }}^{\text {int }}$ is positive, as seen in Fig.2a, suggesting the graphene layer is p-type as its initial polarity. Note that $V_{\text {Dirac }}^{\text {ext }}$ is widely used as a signature of the 
polarity of the graphene layer. However, it can be wrong depending on the extent of the shift voltage, i.e. $V_{\text {Shift }} \equiv V_{\text {Dirac }}^{\text {ext }}-V_{\text {Dirac }}^{\text {int }}$. For example, If $V_{\text {Shift }}>V_{\text {Dirac }}^{\text {ext }}\left(\right.$ i.e. $\left.V_{\text {Dirac }}^{\text {int }}<0\right)$, the graphene layer would be n-type.

To explain this theoretically, a conceptual band diagram is shown in Figs.2c and d. Firstly, Fig.2c shows the impeded electron injection at $V_{G S}=V_{\text {Dirac }}^{\text {int }}$, before $\mathrm{R}_{\text {ext }}$ reaches its peak, in which the injected electron density $\left(\mathrm{n}_{\mathrm{inj}}\right)$ is still less than the injected hole density $\left(\mathrm{p}_{\text {inj }}\right)$ even when $\mathrm{n}=\mathrm{p}=\mathrm{p}_{\text {inj }}$ in the graphene layer at $V_{G S}=V_{\text {Dirac }}^{\text {int }}$. Here, $\mathrm{n}$ and $\mathrm{p}$ are the respective free electron and hole densities within the graphene layer. In contrast, $\mathrm{R}_{\text {ext }}$ at $V_{G S}=V_{\text {Dirac }}^{\text {ext }}$ now exhibits its peak when there is sufficient electron injection due to higher gate bias, resulting in $\mathrm{n}_{\mathrm{inj}}=\mathrm{p}_{\mathrm{inj}}=\mathrm{p}<\mathrm{n}$, as indicated in the conceptual band diagram seen in Fig.2d. Here, a higher gate bias induces more electrons in the channel, making the Schottky barrier narrower at the source contact eluding to higher electron injection $\left(\mathrm{n}_{\mathrm{inj}}\right)$. When $\mathrm{n}_{\mathrm{inj}}$ is balanced with injected holes $\left(\mathrm{p}_{\mathrm{inj}}\right)$, the extrinsic resistance $\left(\mathrm{R}_{\mathrm{ext}}\right)$ exhibits its peak at $V_{G S}=V_{\text {Dirac }}^{\text {ext }}$.

We now extract the free electron (n) and hole (p) densities within the graphene layer from the Hall-effect measurements (see Fig.1e), as a function of $\mathrm{V}_{\mathrm{GS}}$, respectively, using the following relation $^{20-22}$

$$
n, p=\left|\frac{I_{D S} B}{q\left(V_{y 1}-V_{y 2}\right)}\right|,
$$

where $\mathrm{q}$ is the elementary charge. As seen in Fig.2b, the extrapolation lines for electrons and holes, converge at $V_{\text {Dirac }}^{\text {int }}$ rather than at $V_{\text {Dirac }}^{\text {ext }}$. This further confirms that the peak of $\mathrm{R}_{\text {int }}$ is the intrinsic point for $\mathrm{n}=\mathrm{p}$. Here, the slope of the extrapolation line is proportional to the gatecapacitance $\left(\mathrm{C}_{\mathrm{G}}\right)$, as labelled in Fig.2b. 
Using the results in Figs.2a and 2b, a field-effect mobility of the graphene transistor can be retrieved. And there are two types of the field-effect mobility depending on whether the contact effect resides. One of them is the intrinsic carrier mobility $\left(\mu_{\text {int }}\right)$, where the contact effect is removed. And it can be extracted with the following macroscopic definition ${ }^{19}$,

$$
\mu_{\mathrm{int}}=\frac{1}{R_{\mathrm{int}} Q_{n, p}}
$$

where $Q_{n, p}=C_{G}\left|V_{G S}-V_{\text {Dirac }}^{\text {int }}\right|=q n$ or $q p$. Similarly, the extrinsic mobility ( $\left.\mu_{\text {ext }}\right)$ with the contact effect, as the other type, can be defined as $\mu_{\text {ext }}=\left(R_{e x t} Q_{n, p}\right)^{-1}$. Fig.3a shows the extracted $\mu_{\text {int }}$ and $\mu_{\text {ext }}$ for electrons and holes, respectively. As can be seen, $\mu_{\text {int }}$ is higher than $\mu_{\text {ext }}$ In particular, the difference between $\mu_{\mathrm{int}}$ and $\mu_{\mathrm{ext}}$ for electrons is much higher than the case of the hole mobility. This reflects the asymmetrical behaviour of the contact resistance as a function of gate bias, as discussed earlier with Fig.2a. As another observation from Fig.3a, the $\mu_{\text {int }}$ for both electrons and holes is found to be decreased with increasing the gate bias. Since the carrier density is linearly proportional to the gate voltage in the regimes A and B, i.e. $q n, q p=C_{G}\left|V_{G S}-V_{\text {Dirac }}^{\mathrm{int}}\right|$, where each carrier mobility decays (see also Fig.2b), $\mu_{\text {int }}$ can be re-plotted as a function of the carrier density, as shown in Fig.3b. From this, we find that the $\mu_{\text {int }}$ is inversely proportional to the carrier density. This behaviour can be explained with the short-range scattering model ${ }^{23-25}$. This trend is consistent with the expected behavior of a single-layer graphene transistor ${ }^{24,25}$.

In order to explain the intrinsic mobility behaviour, the following microscopic definition of the intrinsic mobility based on the short-range scattering model is employed ${ }^{24}$,

$$
\mu_{\mathrm{int}}=\frac{q v_{F}^{2} \tau_{S}}{\left|E_{F}\right|},
$$



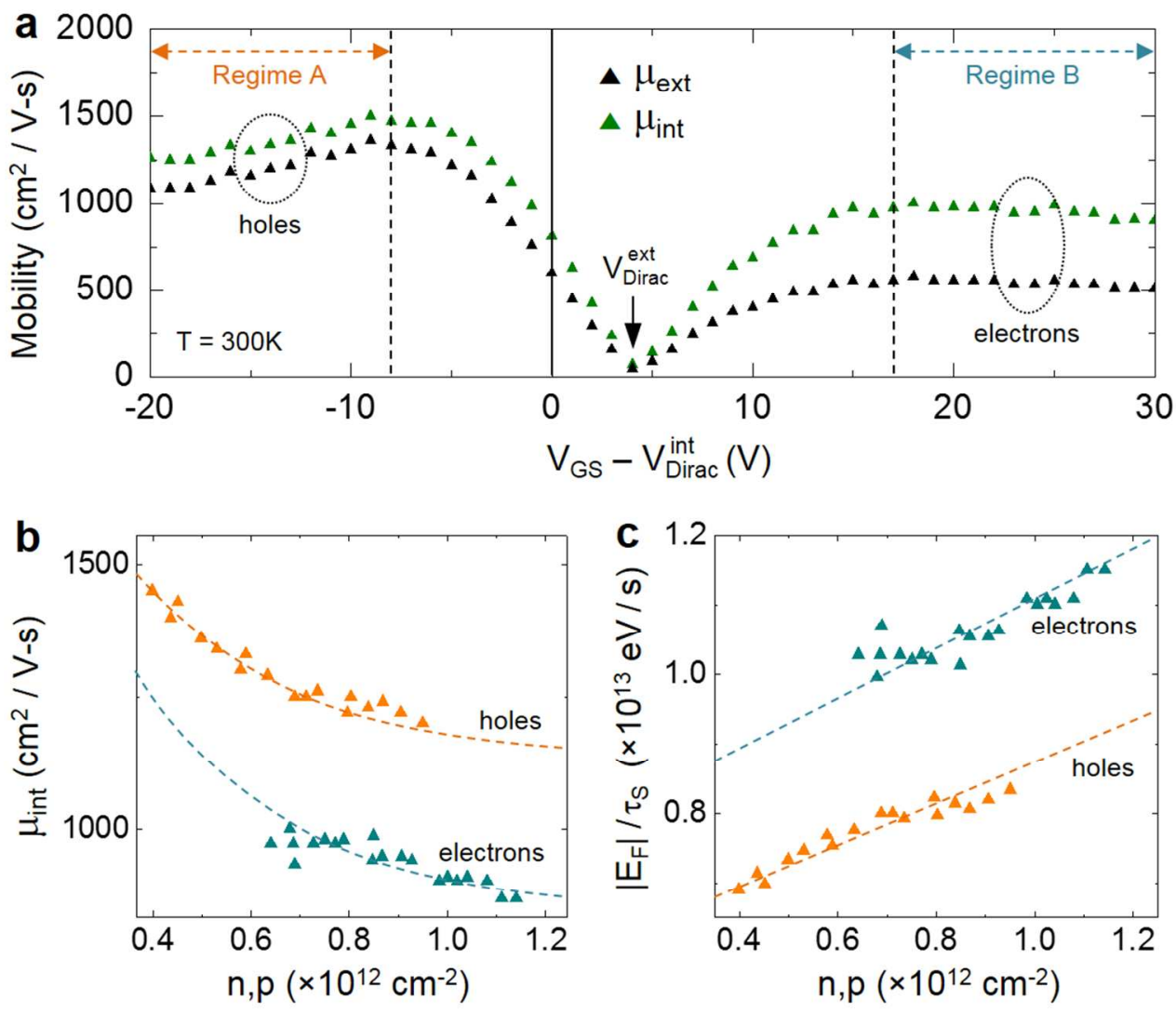

Figure 3. Field-Effect Mobility and Carrier Density: (a) Mobility ( $\mu_{\text {int }}$ and $\mu_{\text {ext }}$ ) as a function of $\mathrm{V}_{\mathrm{GS}}$ calibrated with $V_{\text {Dirac }}^{\text {int }}$. Here, the regime A and B show the mobility decays. (b) Intrinsic mobility $\left(\mu_{\text {int }}\right)$ vs. the carrier density $(n, p)$. (c) Fermi energy per scattering time $\left(\left|E_{F}\right| / \tau\right)$ as a function of the carrier density (n, p). Here, the dotted lines denote the fitted trends.

where $v_{F}$ is the Fermi velocity, $\tau_{\mathrm{S}}$ is the short-range scattering time, and $\mathrm{E}_{\mathrm{F}}$ is the Fermi energy. From Eqs.3 and 4, the carrier density $(n, p)$ is represented as a function of $\left|E_{F}\right| / \tau_{S}$,

$$
n, p=\frac{1}{q^{2} v_{F}^{2} R_{\mathrm{int}}} \frac{\left|E_{F}\right|}{\tau_{S}}
$$

With Eq.5, the correspondence between the carrier density and $\left|\mathrm{E}_{\mathrm{F}}\right| \tau_{\mathrm{S}}$ is computed for $\mathrm{v}_{\mathrm{F}}=10^{8}$ $\mathrm{cm} / \mathrm{s}$, as seen in Fig.3c, clearly confirming their proportionality ${ }^{24,25}$. These results indicate that the dominance of the short-range scattering which is proportional to the carrier density in a single-layer graphene-based field-effect transistor. 
A combination of Hall-effect and Kelvin probe measurements shows a shift in the extrinsic Dirac point from the intrinsic counterpart, suggesting the presence of an electron injection barrier and hence asymmetrical contact resistance as a function of gate bias. This indicates that the Dirac point is shifted by not only the polarity of the graphene layer but also by the contact properties at the source and drain junctions. In addition, the intrinsic carrier mobility is found to be decreased with increasing the gate bias, which is due to an increased short-range scattering of induced carriers in a single layer graphene-based field effect transistor. These results provide analytical physical insight into the correlation between the field-effect parameters and intrinsic material properties.

\section{AUTHOR INFORMATION \\ *Corresponding Author: Arokia Nathan (a299@cam.ac.uk)}

\section{Author Contributions:}

S. L. and A. N. designed the experiment and performed analysis. J. A.-W., P. B.-W., A. A. S., and S. H. prepared the samples. D. H checked the theory and analysis. S. L. and A. N. wrote the manuscript while getting feedback from all other authors.

\section{ACKNOWLEDGMENT}

This work was funded by the Engineering Physical Sciences Research Council (EPSRC), UK as part of Project GRAPHTED (EP/K016636/1). And J. A.-W. acknowledges the support of his Research Fellowship from the Royal Commission for the Exhibition of 1851. All research data are available in the Cambridge University's repository. 


\section{REFERENCES}

1. Semenoff, G. W. Condensed-Matter Simulation of a Three-Dimensional Anomaly. Phys. Rev. Lett. 1984, 53, 2449-2452.

2. Haldane, F. D. M. Model for a Quantum Hall Effect without Landau Levels: condensedmatter realization of the "parity anomaly. Phys. Rev. Lett. 1988, 61, 2015-2018.

3. Novoselov, K. S.; Geim, A. K.; Morozov, S. V.; Jiang, D.; Katsnelson, M. I.; Grigorieva, I. V.; Dubonos, S. V.; Firsov, A. A. Two-Dimensional Gas of Massless Dirac Fermions in Graphene. Nature 2005, 438, 197-200.

4. Zhang, Y.; Tan, Y.-W.; Stormer, H. L.; Kim, P. Experimental Observation of the Quantum Hall Effect and Berry's Phase in Graphene. Nature 2005, 438, 201-204.

5. Castro Neto, A. H.; Guinea, F.; Peres, N. M. R.; Novoselov, K. S.; Geim, A. K. The Electronic Properties of Graphene. Rev. Mod. Phys. 2009, 81, 109-162.

6. Shankar, R. Renormalization-Group Approach to Interacting Fermions. Rev. Mod. Phys. 1994, 66, 129-192.

7. Gorbar, E. V.; Gusynin, V. P.; Miransky, V. A.; Shovkovy, I. A. Magnetic Field Driven Metal-Insulator Phase Transition in Planar Systems. Phys. Rev. B 2002, 66, 045108-1 045108-22.

8. Kotov, V. N.; Pereira, V. M.; Castro Neto, A. H.; Guinea, F. Electron-Electron Interactions in Graphene: Current status and perspectives. Rev. Mod. Phys. 2012, 84, 1067-1125.

9. Du, Y.; Lui, H.; Deng, Y.; Ye, P. D. Device Perspective for Black Phosphorus Field-Effect Transistors: Contact Resistance, Ambipolar Behavior, and Scaling. ACS Nano 2014, 8, 10035-10042.

10. Venugopal, A.; Colombo, L.; Vogel, E. M. Contact Resistance in Few and Multilayer Graphene Devices. Appl. Phys. Lett. 2016, 96, 013512-1 - 013512-3.

11. Xia, F.; Perebeinos, V.; Lin, Y.; Wu, Y.; Avouris, P. The Origins and Limits of MetalGraphene Junction Resistance. Nature Nanotechnology 2011, 6, 179-184.

12. Elias, D. C.; Gorbachev, R. V.; Mayorov, A. S.; Morozov, S. V.; Zhukov, A. A.; Blake, P.; Ponomarenko, L. A.; Grigorieva, I. V.; Novoselov, K. S.; Guinea, F.; Geim, A. K. Dirac Cones Reshaped by Interaction Effects in Suspended Graphene. Nature Physics 2011, 7, 701-704.

13. Blake, P.; Yang, R.; Morozov, S.V.; Schedin, F.; ponomarenko, L.A.; Zhukov, A.A.; Nair, R.R.; Grigorieva, I.V.; Novoselov, K.S.; Geim, A.K. Influence of Metal Contacts and Charge Inhomogeneity on Transport Properties of Graphene near the Neutrality Point. Solid State Commun. 2009, 149, 1068-1071. 
14. Huard, B.; Stander, N.; Sulpizio, J.A.; Goldhaber-Gordon, D. Evidence of the Role of Contacts on the Observed Electron-Hole Asymmetry in Graphene. Phys. Rev. B. 2008, 78, 121402-1 - 121402-4.

15. Zhong, H.; Zhang, Z.; Chen, B.; Xu, H.; Yu, D.; Huang, L.; Peng, L.-M. Realization of Low Contact Resistance close to Theoretical Limit in Graphene Transistors. Nano Research. 2015, 8, 1669-1679.

16. Smith, J. T.; Franklin, A. D.; Farmer, D. B.; Dimitrakopoulos, C. D. Reducing Contact Resistance in Graphene Devices through Contact Area Patterning. ACS Nano 2013, 7, 36613667.

17. Alexander-Webber J. A.; Sagade, A. A.; Aria, A. I.; Van Veldhoven, Z. A.; BrauningerWeimer, P.; Wang, R.; Cabrero-Vilatela, A.; Martin, M.-B.; Sui, J.; Connolly, M. R. Encapsulation of Graphene Transistors and Vertical Device Integration by Interface Engineering with Atomic Layer Deposited Oxide. 2D Mater. 2016, 4, 011008, 1-9.

18. Van Veldhoven Z. A.; Alexander-Webber J. A.; Sagade A. A.; Braeuninger-Weimer P.; Hofmann S. Electronic properties of CVD graphene: The Role of Grain Boundaries, Atmospheric Doping and Encapsulation by ALD. Phys. Status Solidi B 2016, 253, 23212325.

19. S. M. Sze, Physics of Semiconductor Devices (Wiley, Hoboken, NJ, ed. 2, 1981).

20. Tien, D. H.; Park, J.-Y.; Kim, K. B.; Lee, N.; Seo, Y. Characterization of Graphene based FET Fabricated using a Shadow Mask. Sci. Rep. 2016, 6, 25050, 1-8.

21. Xu, H.; Zhang, Z.; Wang, Z.; Wang, S.; Liang, X.; Peng, L.-M. Quantum Capacitance Limited Vertical Scaling of Graphene Field-Effect Transistor. ACS Nano. 2011, 5, 23402347.

22. Chen, B.; Huang, L.; Ma, X.; Dong, L.; Zhang, Z.; Peng, L.-M.; Exploration of Sensitivity Limit for Graphene Magnetic Sensors. Carbon. 2015, 94, 585-589.

23. Sarkar, S.; Amin, K.R.; Modak, R.; Singh, A.; Mukerjee, S.; Bid, A.; Role of Different Scattering Mechanisms on the Temperature Dependence of Transport in Graphene. Sci. Rep. 2015, 5, 16772, 1-10.

24. Zhu, W.; Perepeinos, V.; Freitag, M.; Avouris, P.; Carrier Scattering, Mobilities, and Electrostatic Potential in Monolayer, Bilayer, and Trilayer Graphene. Phys. Rev. B 2009, 80, 235402-1 - 235402-8.25.

25. Hwang, E. H.; Adam, S.; Das Sarma, S.; Carrier Transport in Two-Dimensional Graphene Layers. Phys. Rev. Lett. 2007, 98, 186806-1 - 186806-4. 

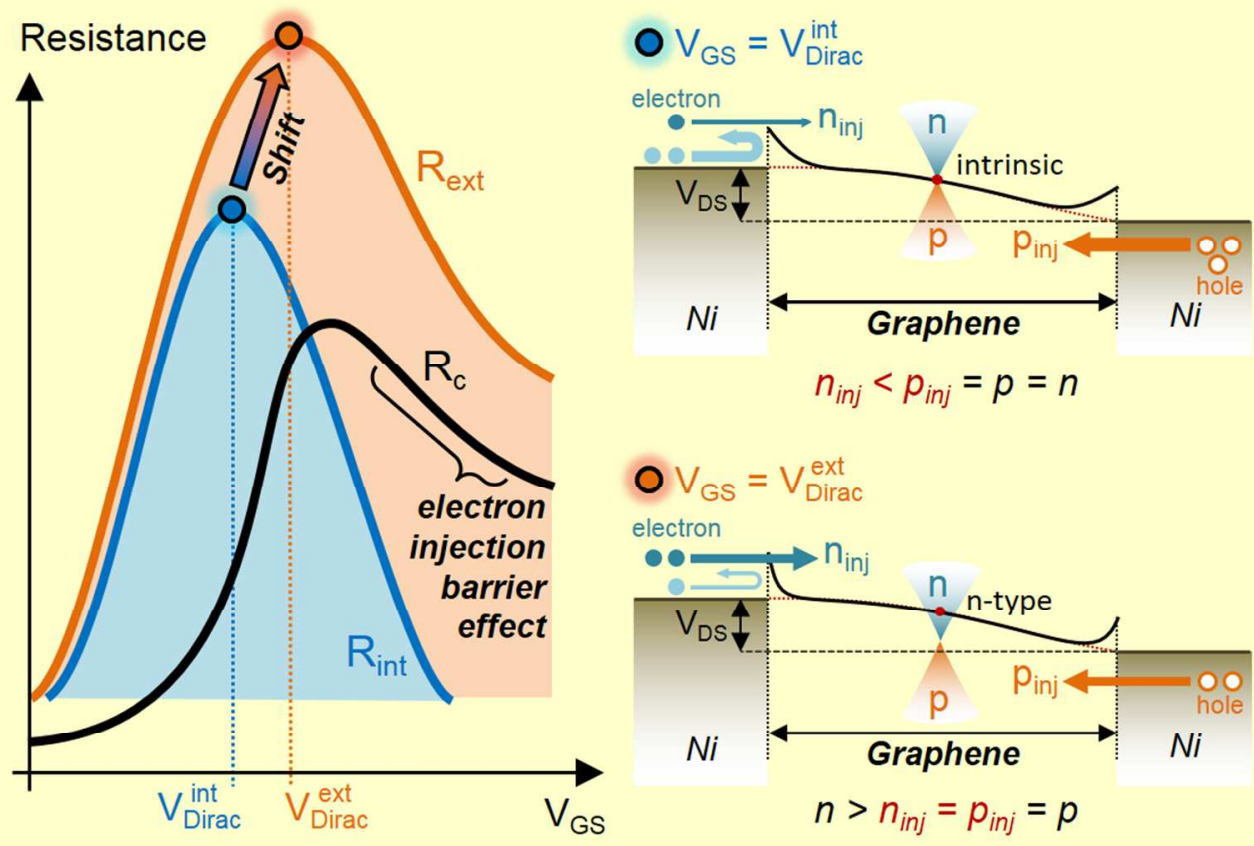

$$
n_{i n j}<p_{i n j}=p=n
$$

$\mathrm{O} V_{G S}=V_{\text {Dirac }}^{\text {ext }}$ electron

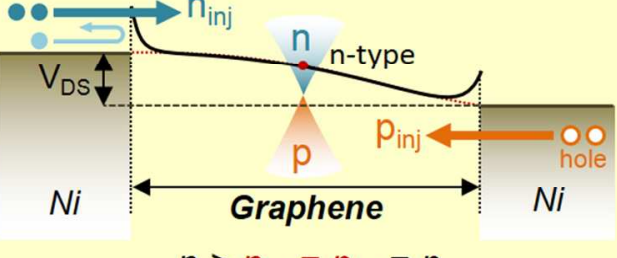
$n>n_{i n j}=p_{i n j}=p$

$97 \times 64 \mathrm{~mm}(300 \times 300$ DPI $)$ 